\title{
A Novel Approach to Model the Thermal-electrical Behavior of Pyroelectric Infrared Sensors
}

\author{
Robin Lehmkau' ${ }^{1}$, Jens Lienig ${ }^{2}$ \\ 1 InfraTec GmbH, Gostritzer Str. 61, 01217 Dresden (Germany), \\ 2 TU Dresden, Institute of Electromechanical and Electronic Design, 01062 Dresden (Germany) \\ r.lehmkau@infratec.de
}

\begin{abstract}
Summary:
The combined evaluation of the thermal and electrical domain of a pyroelectric system is a challenging task. The proposed approach precisely models the thermal system with FEM. The output data is approximated with a fit function and transferred to SPICE creating a universal and adaptable model for the whole pyroelectrical signal chain. For validation, the InfraTec detector LRM-244 is used.
\end{abstract}

Keywords: FEM, SPICE, pyroelectric detector, thermal-electrical model, LRM-244

\section{Motivation}

Pyroelectric detectors are used for high-performance contactless gas analysis and fast flame detection. To accelerate the design and development process of improved detectors with new materials or geometry, a precise and customizable model is needed. The main dependencies of the generated pyroelectric current $I_{\text {pyro }}$ are the pyroelectric coefficient $p$ of the material, the effective surface $A$ and the time derivation of the temperature change $\mathrm{d} T / \mathrm{d} t$ shown in Eq. (1).

$$
I_{\text {pyro }}=p \cdot A \cdot \frac{\mathrm{d} T}{\mathrm{~d} t} \quad \text { Eq. (1) }
$$

The prediction of the temperature behavior is a challenging task. Specifically, the transfer of the temperature data to the electrical domain in order to evaluate the resulting pyroelectric current and output voltage are an open problem.

The contribution of this paper is an optimized approach to model the signal behavior of pyroelectric detectors regarding the whole signal chain from the incident thermal radiation to the electrical output signal. The method can be used for any detector geometry, pyroelectric material, and electrical readout circuit.

\section{Thermal System}

Often, the thermal behavior is modeled by a simple low-pass consisting of a thermal resistance $R_{\text {th }}(\mathrm{K} / \mathrm{W})$ and a thermal capacitance $C_{t h}(\mathrm{~J} / \mathrm{K})$. An analytical method is to solve the equation for the one-dimensional thermal conduction, by which the temporal and additionally spatial resolution can be analyzed [1].

A more precise approach uses several RC-elements building the dominating conducting paths of a thermal system, which can be connected like a possibly multidimensional Cauer or Foster ladder as shown in Fig. 1. Note that inaccuracies of this model can occur due to the limited number of lumped RC-elements [2].

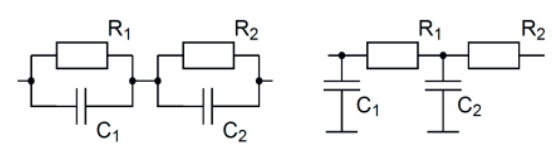

Fig. 1: Foster- (left) and Cauer (right) ladder of a simple thermal system

The most precise solution is achieved by using a software with the finite-element-method (FEM). Moreover, any detector shape can be modeled fast with CAD. It is helpful to define an interface between the thermal and electrical subdomains, as the calculation of a multiphysics problem with FEM needs much setup and computing effort.

Therefore, we simulate the thermal system with the FEM-tool COMSOL Multiphysics ${ }^{T M}$. The pyroelectric detector LRM-244 of InfraTec serves as demonstration example. It is sufficient to model the sensor chip, the absorption layer, the optical filter, glue dots, the gold coated chip holder and a small part of the circuit board surrounded by air, illustrated for one signal channel in Fig. 2. We noticed further details can be neglected.

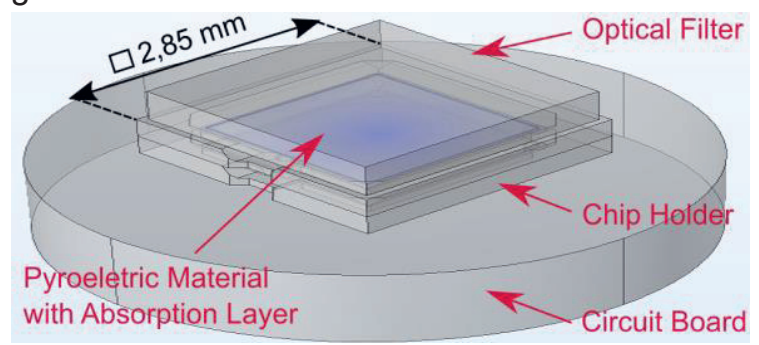

Fig. 2: FEM model of LRM-244 with the thermal dominating parts. The surrounding air block is hidden. 
The input signal is modeled as thermal flux with the property "thermal perturbation" and fed into the absorption layer. The outer faces of the circuit board are kept at ambient temperature. As result the temperature change $\Delta T(f)$ of the pyroelectric material depending on discrete steps of the excitation frequency can be investigated.

\section{Thermal-electrical Interface}

The next step is to fit a continuous regression function $A(s)$ to the simulated discrete behavior $\Delta T(f)$. Simplified pyroelectrical systems have two dominating thermal time constants, depending on the sensitive material itself $T_{1}$ and the absorption layer $T_{2}$. They each can be described by a classical first order low-pass in Laplace notation with the complex frequency parameter $s$. Besides, there can be deviations from a typical lowpass behavior due to a special detector geometry or additional material layers. That is why $A(s)$ in Eq. (2) is extended by the terms containing $T_{3}$ and $T_{4}$ to support the precise evolving of the regression function.

$$
A(s)=v \cdot \frac{1}{1+s^{\cdot} \tau_{1}} \cdot \frac{1}{1+s^{\cdot} \tau_{2}} \cdot \frac{1+s^{\cdot} \tau_{3}}{1+s^{\cdot} \tau_{4}}
$$

Here, the variable $v$ is a linear conversion factor considering the used incident radiation flux of $1 \mathrm{~W} / \mathrm{m}^{2}$ in FEM. The parameters of $A(s)$ are calculated by a function of SciPy using the leastsquare-method. A weigh function is implemented to ensure a small relative error over the relevant frequency range from typically $0,1 \mathrm{~Hz} \ldots 1 \mathrm{kHz}$.

Figure 3 shows the fit function of the thermal system compared to a simple first order low-pass with $T_{t h}=150 \mathrm{~ms}$. Especially at higher modulation frequencies, the relative error between simulation and fit stays below $0,5 \%$ for $f<10 \mathrm{kHz}$.

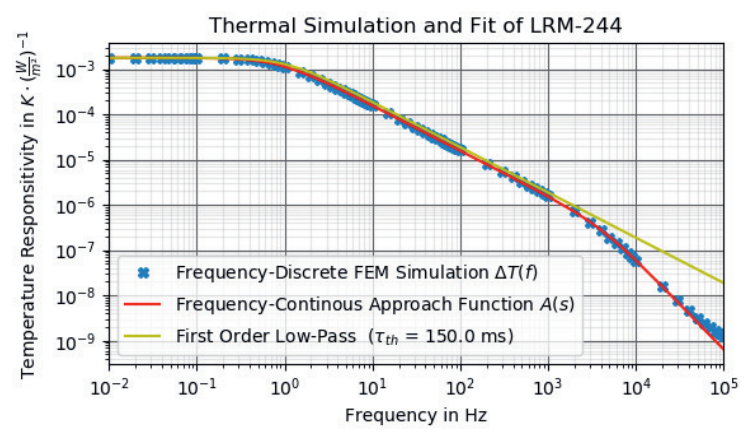

Fig. 3: Comparison of the discrete simulation data, continuous fit function and a first order low-pass

\section{Simulation of the Pyroelectrical System}

The thermal fit function $A(s)$ can be transferred to a circuit analysis program like LTSpice using an arbitrary behavioral voltage source $V_{1}$. Referring to Eq. 1, the time derivate of the temperature change can be simulated using $V_{2}$. Finally, a voltage dependent current source $I_{1}$ with the gain factor " $p \cdot A$ " generates the frequency dependent pyroelectric current for an input radiation $\Phi(t)$.

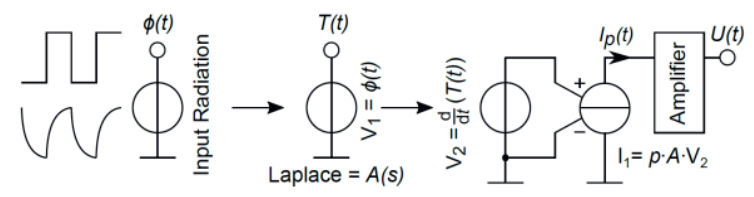

Fig. 4: Signal chain of the pyroelectrical system

The current $I_{\mathrm{p}}(t)$ is fed into an electrical amplification circuit. For the LRM-244, a transimpedance amplifier with $100 \mathrm{G} \Omega$ feedback is used.

\section{Model Validation}

The results of the novel approach are demonstrated in Fig. 5. The relative errors of noise and relative amplitude between simulation and measurement are typically below $10 \%$ and deviations mostly depend on the thermal and electrical component tolerances.
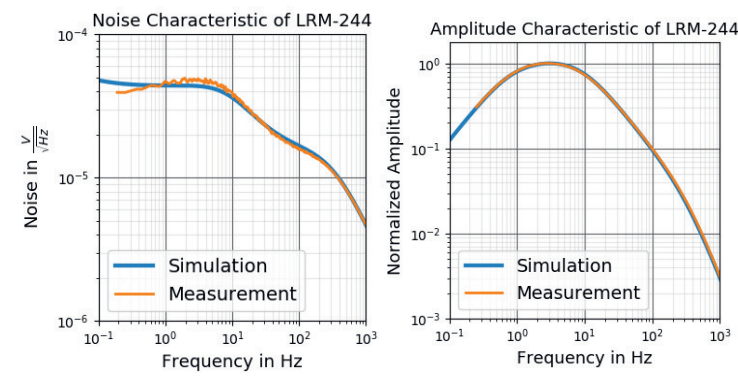

Fig. 5: Noise (left) and amplitude (right) characteristic of LRM-244 compared to the measured signals

The following table shows the results of an absolute signal simulation compared to a measurement with a lab setup and blackbody emitter (500 K, $10 \mathrm{~Hz}$, no filter).

\begin{tabular}{|c|c|c|c|}
\hline Intensity & Simulation & Measurement & Rel. Error \\
\hline $3,26 \mathrm{~W} / \mathrm{m}^{2}$ & $1,15 \mathrm{~V}_{\mathrm{rms}}$ & $1,27 \mathrm{~V}_{\mathrm{rms}}$ & $9,4 \%$ \\
\hline
\end{tabular}

\section{Conclusion}

The proposed new model for pyroelectric systems offers a fast and precise prediction of the signal and noise behavior of any detector geometry. Both the thermal and the electrical system can be largely adapted and combined with arbitrary material parameters. Even in early stages of the design process, the frequency dependent signal-to-noise-ratio (SNR) and the specific detectivity $D^{*}$ can be assessed and optimized.

\section{References}

[1] H. Budzier, G. Gerlach: "Thermische Infrarotsensoren: Grundlagen für Anwender" WileyVCH (2010), ISBN: 978-3-527-40960-0

[2] M. Touzelbaev et al.: "High-Efficiency Transient Temperature Calculations for Applications in Dynamic Thermal Management of Electronic Devices" Journal of Electronic Packaging (2013), DOI: $10.1115 / 1.4024747$ 